\title{
IL-27 regulates the adherence, proliferation, and migration of MSCs and enhances their regulatory effects on Th1 and Th2 subset generations
}

\author{
Fenghuang $\mathrm{Xu}^{1} \cdot$ Junzhu $\mathrm{Yi}^{1} \cdot$ Zhuoya Wang $^{1} \cdot$ Yejia $\mathrm{Hu}^{2} \cdot$ Chunlei Han $^{3} \cdot$ Qun Xue $^{4}$. \\ Xueguang Zhang ${ }^{4} \cdot$ Xiying Luan ${ }^{1,5}$
}

Published online: 14 June 2017

(C) The Author(s) 2017. This article is an open access publication

\begin{abstract}
Interleukin 27 (IL-27) regulates T cell function and is involved in inflammation. It has been reported that human placenta-derived mesenchymal stromal cells (hPMSCs) can inhibit $\mathrm{T}$ cell responses and attenuate inflammation reactions. However, it is unclear whether IL27 can regulate hPMSC function. Here, we examined the effects of IL-27 upon adherence, migration, and proliferation as well as the immunomodulatory effects of hPMSCs. The results show that IL-27 receptor $\alpha$ chain $(\mathrm{IL}-27 \mathrm{R} \alpha)$ is expressed in hPMSCs. IL-27 at $30 \mathrm{ng} / \mathrm{ml}$ inhibited hPMSC adherence and proliferation, while the migration of hPMSCs was promoted with IL-27 at doses of 20 or $30 \mathrm{ng} / \mathrm{ml}$, as determined with use of real-time cell analysis (RTCA). Moreover, IL-27 promoted regulatory effects of hPMSCs through enhancing Th2 and suppressing Th1 subset generation from activated $\mathrm{T}$ cells in human peripheral blood. IL-27 also enhanced the ability of hPMSCs to secrete IL-10 from $\mathrm{CD}^{+} \mathrm{T}$ cells through increased expression levels of the programmed death ligand
\end{abstract}

Fenghuang Xu and Junzhu Yi contributed equally to this work.

Xiying Luan

xyluan@sohu.com

1 Department of Immunology, Binzhou Medical University, Yantai, Shandong Province 264003, People's Republic of China

2 Department of Pathophysiology, Binzhou Medical University, Yantai, Shandong Province 264003, People's Republic of China

3 Department of Health Statistics, Binzhou Medical University, Yantai, Shandong Province 264003, People's Republic of China

4 Medical College of Soochow University, Suzhou, Jiangsu 215006, People's Republic of China

5 Taishan Scholar Immunology Program, Binzhou Medical University, Yantai, Shandong Province 264003, People's Republic of China
1 (PDL1) in hPMSCs via the Janus kinase (JAK)/signal transducer and activator of transcription 1 (STAT1) signaling pathway. In conclusion, IL-27 has significant modulatory effects on adherence, proliferation, and migration of hPMSCs. IL-27 increased PDL1 expression levels in hPMSCs via the JAK/STAT1 pathway, which then enhanced the regulatory effects of hPMSCs upon Th1 and $\mathrm{Th} 2$ cell generations and IL-10 secretion from $\mathrm{CD} 4^{+} \mathrm{T}$ cells.

Keywords IL-27 · Mesenchymal stromal cells (MSCs) · Th1 $\cdot$ Th2 $\cdot$ IL-10 $\cdot$ Programmed death ligand 1 (PDL1)

\section{Introduction}

Interleukin 27 (IL-27) is a type-I-cytokine of the IL-12 cytokine superfamily and is predominantly secreted by macrophages and dendritic cells. The receptor for IL-27, which is expressed in T and B lymphocytes, is composed of a gp130 and IL-27 receptor $\alpha$ chain (the IL-27R $\alpha$ is also known as WSX1 or TCCR) [1-3]. IL-27R $\alpha$ and gp130 are expressed in human placenta tissue and bone marrow-derived mesenchymal stromal cells $[4,5]$; however, little information is available regarding the expression of IL-27R $\alpha$ in human placentaderived mesenchymal stromal cells (hPMSCs). IL-27 has been shown to be involved with regulating the generation of T cell subsets. For example, IL-27, which can act as a proinflammatory cytokine to promote T-bet activation, then induces IL-12R $\beta 2$ expression in T cells. Moreover, IL-27 can stimulate the differentiation of $\mathrm{T}$ cells into its subset, Th1, which can then promote the progress of inflammation [6]. IL-27 plays a pro-inflammatory role in graft versus host disease (GVHD) biology, as blocking of IL-27 signaling reduced GVHD in mice through augmenting Treg reconstitution [7]. 
IL-27 can also exert anti-inflammatory effects, through inhibiting the generation of T cells into its subset Th17 via signal transducer and activator of transcription 1 (STAT1) and STAT3 signaling pathways, thus alleviating the symptoms of experimental autoimmune encephalomyelitis (EAE) [1,8]. Collating the information from these studies leads to the conclusion that IL-27 regulates immune function in infectious and autoimmune diseases via intervening with $\mathrm{T}$ cell subset transformation [9].

Results from numerous studies have demonstrated that mesenchymal stromal cells (MSCs) can be used in the treatment of inflammatory and autoimmune diseases [10]. Following tissue damage, MSCs migrate directly to damaged areas, where they regulate the activity and function of various immune cells to gradually reduce tissue damage and stimulate the secretion of growth factors which promote tissue repair by enhancing the differentiation of tissue precursor cells [11]. Moreover, inflammatory factors located at the sites of damage, such as interferon (IFN)- $\gamma$ and tumor necrosis factor (TNF)- $\alpha$, can, in turn, influence the biological function of MSCs. Zhang et al. reported that long-term IFN- $\gamma$ could inhibit MSC proliferation [12]; and increased TNF- $\alpha$ secretion in systemic lupus erythematosus (SLE) serum significantly inhibited the migration and in vivo homing capacities of SLE bone marrowderived MSCs (BMSCs) [13]. However, it is not clear whether IL-27 in these inflammatory reactions affects the activities of MSCs by regulating their proliferation, migration, and/or other biological functions of MSCs.

The immunomodulatory effects of MSCs on T cells have been observed in several paradigms. For example, MSCs can inhibit $\mathrm{T}$ cell proliferation and secretion of the Th1 cytokine IFN- $\gamma$, as well as induce the generation of the T cell subset $\mathrm{CD} 4^{+} \mathrm{CD} 25^{+} \mathrm{Foxp}^{+}{ }^{+}$Treg; however, the underlying mechanisms of these processes are not known $[14,15]$. In addition, cytokines produced by activated $\mathrm{T}$ cells can also regulate MSCs [16]. Previous work from our laboratory has revealed that IFN- $\gamma$ and TNF- $\alpha$ enhance the capacity for hPMSCs to induce the differentiation of activated $\mathrm{T}$ cells to IL- $10^{+} \mathrm{T}$ cell subsets and, in this way, exert a remodeling effect upon activated T cells [17]. Moreover, IFN- $\gamma$ can enhance MSCs ability to inhibit $\mathrm{T}$ cell proliferation by upregulating the expression of the programmed death ligand 1 (PDL1) in MSCs [18-20]. However, it is unclear whether IL-27 can affect T cell differentiation into Th1 or Th2 subsets by regulating PDL1 expression in hPMSCs.

In the current study, we first investigated the expression of IL-27R $\alpha$ in hPMSCs, and then investigated the effects of IL27 on adherence, migration, and proliferation of hPMSCs, as well as on the regulatory effects of hPMSCs on Th1 or Th2 cell generation. The significance of this study is its potential to identify the benefits of hPMSCs for using in clinical cell therapy.

\section{Materials and methods}

\section{Isolation of hPMSCs}

The hPMSCs used in this study have been isolated as described previously [21]. Briefly, placenta were obtained from healthy women who delivered after a full-term pregnancy. After the decidua tissue on placenta was removed and placenta was washed in D-Hanks solution, they were sectioned into small fragments and homogenized. The tissues were then incubated with $0.1 \%$ IV collagenase (Gibco, CA, USA) for $30 \mathrm{~min}$ at $37^{\circ} \mathrm{C}$, followed by filtering through a screen filter (100 mesh). After centrifugation at $524 \times \mathrm{g}$ for $10 \mathrm{~min}$, cells were washed with D-Hanks solution, counted, and then incubated at $37{ }^{\circ} \mathrm{C}$ in a $5 \% \mathrm{CO}_{2}$ environment. The cells were passaged once every 7-8 days with half of the medium replaced with new medium on day 3 . The hPMSCs were identified by the following: (1) cell morphology as observed using microscopy, (2) the detection of cell surface antigens (CD105, CD73, CD90, CD34, CD14, CD19, and human leukocyte antigen-antigen D related (HLA-DR)) as determined using flow cytometry (FCM), and (3) the ability to differentially detect between bone and fat cells. Identified hPMSCs were used in the experiment after three passages. The project was approved by the Ethics Committee of the Affiliated Hospital of Binzhou Medical College, Yantai, and informed consent was obtained from all sample donors.

\section{Adipogenic and osteogenic inductions}

HPMSCs were seeded in six plates for adipogenic and osteogenic induction. The hPMSCs reached $70 \%$ and $100 \%$ confluency for adipogenic and osteogenic induction, respectively. The medium was removed and then cultured with adipogenic and osteogenic differentiation medium. All differentiation processes were in strict accordance with the kit instructions (Wei Tong Biotechnology, China). Cells cultured without adipogenic or osteogenic differentiation medium were used as negative controls for adipogenic and osteogenic differentiation. For adipogenic staining, cells cultured with or without adipogenic differentiation medium were stained with Oil Red $\mathrm{O}$ after 14 days. For osteocyte staining, cells cultured with or without osteogenic differentiation medium were stained with Alizarin Red after 28 days.

\section{PBMC isolation}

Peripheral blood mononuclear cells (PBMCs) were isolated from whole blood as described previously [22]. Briefly, the blood was obtained from healthy adults at the Central Blood Bank in Yantai City. Informed consent was acquired from all donors. After being anti-coagulated and diluted with an equal volume of D-Hanks solution, the blood samples were added to 
Ficoll separating medium. The PBMC suspension was prepared using a density gradient centrifugation method.

\section{RT-PCR analysis}

Using $\mathrm{CD}^{+}{ }^{+} \mathrm{T}$ cells, which expressed IL-27R $\alpha$ and served as a positive control and LNCaP cells as a negative control [23, 24], the messenger RNA (mRNA) expression of IL-27R $\alpha$ in hPMSCs was detected using RT-PCR. Total RNA was extracted using TRIzol (Invitrogen, CA, USA). RNA was then transcribed into complementary DNA (cDNA) using the Revert Aid First Strand cDNA Synthesis Kit (Thermo Scientific, CA, USA) according to the operating instructions. PCR reactions were conducted using the $2 \times$ Taq PCR Master Mix Kit (Thermo Scientific, CA, USA). The primer sequences were as follows: IL-27R $\alpha-5^{\prime}$-ACC CAA ATG AAG CCA AAC GC-3', 5'-CGC CCC ACA AAT CCT CTT CT-3'; $\beta$-actin5'-GGC ACC CAG CAC AAT GAA-3', 5'-GGA AGG TGG ACA GCG AGG -3'. PCR reaction conditions included $30 \mathrm{cy}-$ cles at $94{ }^{\circ} \mathrm{C}$ for $2 \mathrm{~min}, 94^{\circ} \mathrm{C}$ for $30 \mathrm{~s}, 55^{\circ} \mathrm{C}$ for $30 \mathrm{~s}$, and $72{ }^{\circ} \mathrm{C}$ for $1 \mathrm{~min}$, followed by $72{ }^{\circ} \mathrm{C}$ for $5 \mathrm{~min}$. PCR products were analyzed using $1 \%$ agarose gel electrophoresis.

\section{Gene sequencing}

Gene sequencing was conducted for IL-27R $\alpha$ mRNA in hPMSCs. A portion of the PCR products, as generated using procedures described above, was gene sequenced at the Shanghai Meiji Biomedical Co., Ltd. The gene sequencing results were compared with those of the National Center for Biotechnology Information (NCBI) using the Blast program (http: //www.ncbi.nlm.nih Gov/BLAST).

\section{Western blot analysis}

Protein levels of IL-27R $\alpha$ in hPMSCs were determined using Western blot with $\mathrm{CD}^{+} \mathrm{T}$ cells serving as a positive control. Expressions of IL-27R $\alpha$ in hPMSCs as determined on different culture days for one generation were then analyzed by Western blot, as were levels of phosphorylated STAT1 (PSTAT1) and STAT1 in these hPMSCs. HPMSCs were pretreated with the Janus kinase $1 / 2$ (JAK1/2) inhibitor INCB018424 (20 ng/ml, Selleck, Shanghai, China) for $1 \mathrm{~h}$ before stimulation with IL-27 and incubated in the presence or absence of INCB018424 for an additional $1 \mathrm{~h}$; P-STAT1 and STAT1 expressions were then measured by means of Western blot. After adding RIPA lysis buffer to hPMSCs, the cells were lysed on ice for $40 \mathrm{~min}$, centrifuged, subjected to SDS-PAGE electrophoresis, and then transferred to PVDF membranes. Rabbit anti-human IL-27R $\alpha$ (Bioss, Beijing, China), $\beta$-actin (Bioworld, Nanjing, China), phosphorylated STAT1 (Abcam, Cambridge, UK), or STAT1 (Proteintech, Wuhan, China) antibodies were incubated with membranes at $4{ }^{\circ} \mathrm{C}$ overnight. Secondary goat anti-rabbit antibody (1: 1000) (Santa Cruz, CA, USA) was added on the following day. Blots were further washed and developed with enhanced chemiluminescent substrate (Beyotime, Shanghai, China), and protein bands were then visualized using a Western blot imager.

\section{hPMSC adherence and proliferation}

The hPMSC adherence and proliferation were analyzed with real-time cell analysis (RTCA) using the xCELLigence system E-Plate. Dulbecco's modified essential medium (DMEM) supplemented with $10 \%$ fetal bovine serum (FBS) was added to the E-Plate, and the background cell index (CI) values were then recorded. The hPMSC suspension (10,000/well) was then added to the medium followed by IL-27 (Peprotech, NJ, USA) with final concentrations of $0,10,20$, or $30 \mathrm{ng} / \mathrm{ml}$. The hPMSCs were then incubated, and cell adherence and proliferation were observed at $5 \mathrm{~min}$ intervals using the RTCA analyzer. CI values were recorded, and adherence and proliferation were observed continuously for 6 and $80 \mathrm{~h}$, respectively. The rate of cell growth was calculated based on the slope of the line between two given time points [25].

\section{hPMSC migration}

RTCA was used to monitor the migration of hPMSCs in realtime as determined with use of the xCELLigence system CIM-plates. Medium containing 10\% FBS (165 $\mu \mathrm{l} /$ well) was added to the lower chamber of the CIM-plates, while $30 \mu \mathrm{l} /$ well DMEM was added to the upper chamber. After incubation for $1 \mathrm{~h}$, baseline background levels were recorded. The hPMSC (40,000/well) suspensions without FBS were added to the upper chamber, followed by IL-27 at final concentrations of $0,10,20$, or $30 \mathrm{ng} / \mathrm{ml}$. RTCA was recorded at 5 min intervals. CI values and real-time dynamic analysis of migration were recorded over a $24 \mathrm{~h}$ period.

\section{Co-culturing hPMSCs and PBMCs}

The 24-well plates containing hPMSCs $\left(5 \times 10^{4}\right.$ cells/well $)$ were treated with IL-27 $(20 \mathrm{ng} / \mathrm{ml})$ for $24 \mathrm{~h}$. Then, PBMC $\left(5 \times 10^{5}\right.$ cells/well) and PHA $(10 \mu \mathrm{g} / \mathrm{ml})$ (Solarbio, Beijing, China) were added [26]. PBMC and PBMC with PHA were used as negative and positive controls, respectively. After incubation for $72 \mathrm{~h}$, PBMCs were analyzed using FCM analysis.

\section{PDL1 mAb blocking test}

After incubation for $24 \mathrm{~h}$, mouse anti-human PDL1 monoclonal antibody (mAb) $(5 \mu \mathrm{g} / \mathrm{ml})$ (BioLegend, CA, USA) was added to hPMSCs [26], followed by PBMC and 
PHA. PBMCs were then collected $72 \mathrm{~h}$ later and analyzed with use of FCM.

\section{Flow cytometry analysis}

FCM was used to assess the regulatory effects of IL-27 on PDL1 expression in hPMSCs. The hPMSCs were cultured in 24-well plates for $24 \mathrm{~h}$ followed by the addition of varying concentrations of IL-27 $(0,10,20$, or $30 \mathrm{ng} / \mathrm{ml})$. The hPMSCs were collected at $12,24,48$, or $72 \mathrm{~h}$ post-IL-27 treatment. Phycoerythrin (PE)-labeled mouse anti-human PDL1 mAb (Ebioscience, CA, USA) were then added and incubated under dark conditions at $4{ }^{\circ} \mathrm{C}$ for $30 \mathrm{~min}$. FCM analysis was then performed.

Membrane and intracytoplasmic molecules in T cells were detected by FCM. For intracellular staining, PBMCs were fixed, permeabilized, and stained with fluorescein isothiocyanate (FITC)-labeled mouse anti-human IFN- $\gamma$ mAb, PElabeled mouse anti-human IL-4 mAb, and IL-10 mAb. T cell surface molecules were stained with allophycocyanin (APC)labeled mouse anti-human CD3 mAb, peridiinin-chorophyll protein complex (PerCP)-labeled mouse anti-human CD4 mAb (Miltenyi Biotec, Bergisch Gladbach, Germany). HPMSC surface molecules were stained with FITC-labeled mouse anti-human CD14 mAb, CD34 mAb, CD90 mAb, CD105 mAb, HLA-DR mAb, and PE-labeled mouse antihuman CD19 mAb, CD73 mAb (Miltenyi Biotec, Bergisch Gladbach, Germany), IL-27R $\alpha$ mAb (R\&D Systems, USA) at $4{ }^{\circ} \mathrm{C}$ for $30 \mathrm{~min}$, then washed and analyzed with FCM. As negative controls for the fluorescent cell labeling, we employed appropriate isotype controls for the antibodies employed (Miltenyi Biotec, Bergisch Gladbach, Germany). All mAbs used were at concentrations recommended by the manufacturer.

\section{Statistical analysis}

Data were expressed as mean \pm standard deviation. All data were analyzed with SPSS20 statistical software. Differences among groups were calculated using ANOVA, and multiple comparisons were performed using the Bonferroni correction. A $P<0.05$ was required for results to be considered statistically significant.

\section{Results}

\section{Phenotypic characteristics and differentiation of hPMSCs}

To verify that the cells separated and cultured were hPMSCs, the phenotype of hPMSCs was determined with use of FCM. The results showed that about $95 \%$ of the isolated hPMSCs expressed CD73 and CD90 and CD105, but not CD14, CD19, CD34, or HLA-DR (Fig. 1a). The hPMSCs cultured showed a fibroblast-like appearance and a capacity for attachment to plastic (Fig. 1b).

To determine whether hPMSCs could differentiate into multiple mesenchymal cell lineages, hPMSCs were cultured in adipogenic and osteogenic medium, respectively. In the adipocyte induction medium, adipocytes were verified by the presence of Oil Red O staining of fat globules (Fig. 1d). In the osteoblast induction medium, Alizarin red staining showed intracellular calcium deposits (Fig. 1f). As no fat globules and intracellular calcium deposits were found in hPMSC control groups which were cultured with DMEM supplemented with $10 \%$ FBS, these results indicated that the cells were, in fact, hPMSCs (Fig. 1c, e).

\section{Expression of IL-27R $\alpha$ in hPMSCs}

We first found that hPMSCs expressed IL-27R $\alpha$ in gene and protein levels. IL-27R $\alpha$ mRNA was expressed in hPMSCs as revealed with use of RT-PCR (Fig. 2a). To further corroborate these RT-PCR results, IL-27R $\alpha$ PCR products were sequenced (Fig. 2b) and found to match with that of the 503-789 bp of IL-27R $\alpha$ in the NCBI gene bank (Fig. 2c). Results obtained from Western blot and FCM also demonstrated that IL- $27 \mathrm{R} \alpha$ protein was expressed in hPMSCs (Fig. 2d). As shown in Fig. 2e, levels of IL-27R $\alpha$ in hPMSCs were not the same in different culture times, with maximal expressions of IL$27 \mathrm{R} \alpha$ being obtained on the seventh day of culture in passage three $(P<0.01$; Fig. 2e, f).

\section{IL-27 regulates the adherence, migration, and proliferation of hPMSCs}

On the basis of above results, we next investigated the effects of IL-27 on hPMSC adherence, migration, and proliferation. Our results indicated that IL-27 can regulate the adherence, migration, and proliferation of hPMSCs (Fig. 3). IL-27 at $30 \mathrm{ng} / \mathrm{ml}$ inhibited hPMSC adherence $(P<0.05$; Fig. 3a, b) as compared to controls, and incubation with $30 \mathrm{ng} / \mathrm{ml}$ of IL27 for 2,4 , or $6 \mathrm{~h}$ resulted in significantly lower adherent CI values as compared to that of controls $(P<0.05$; Fig. $3 \mathrm{c})$. IL27, at concentrations of 20 and $30 \mathrm{ng} / \mathrm{ml}$, promoted hPMSC migration $(P<0.05$; $P<0.01$; Fig. 3d, e). Significant increases in CI values for hPMSC migration as compared to controls were observed when IL-27 (20 or $30 \mathrm{ng} / \mathrm{ml})$ was incubated for 6, 12, or $24 \mathrm{~h}(P<0.05$; $P<0.01$; Fig. 3f). Proliferation of hPMSCs was significantly inhibited by IL27 at $30 \mathrm{ng} / \mathrm{ml}$, but not at 10 or $20 \mathrm{ng} / \mathrm{ml}$ as indicated by CI values obtained within the $80 \mathrm{~h}$ of testing and changes in the slope of the curve between the 0 and $80 \mathrm{~h}$ periods $(P<0.05$; Fig. 3g-i). 
Fig. 1 Immunophenotyping and differentiating capabilities of hPMSCs. Phenotypic characterization of hPMSCs by FCM analysis (a). Isotype controls are presented as green histograms. The red line in each histogram represents the specific expression of the indicated cell surface marker. The morphology of hPMSCs from three passages (b). Multilineage differentiation potential was assessed by their capacity to differentiate into adipocytes as stained by Oil Red $\mathrm{O}$ (d) and osteoblasts as stained by Alizarin red staining (f). Oil Red $\mathrm{O}(\mathbf{c})$ and Alizarin red (e) staining of hPMSCs as control (color figure online) a
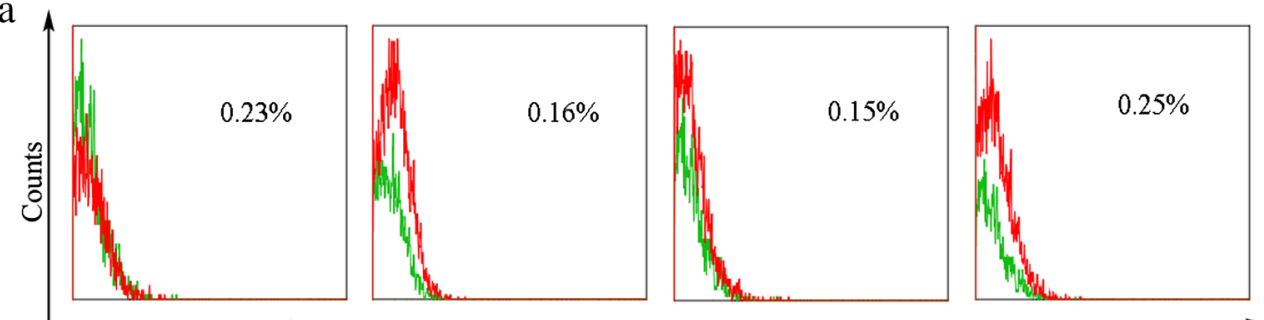

CD14-FITC

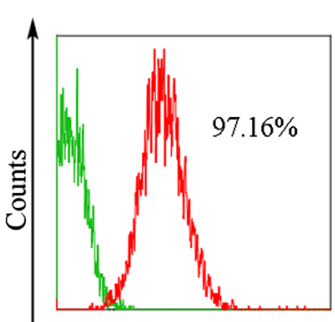

CD19-PE

CD34-FITC

$\mathrm{b}$

HLA-DR-FITC

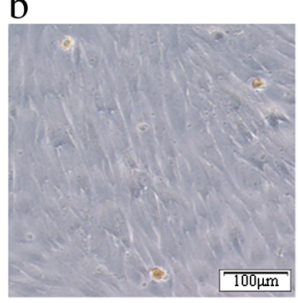

c
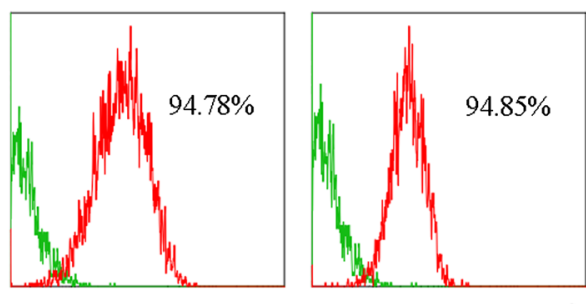

CD105-FITC

d

e
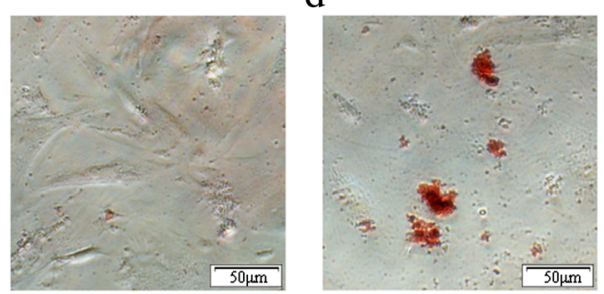

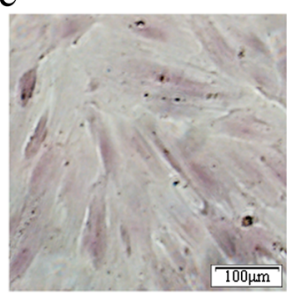

$\mathrm{f}$

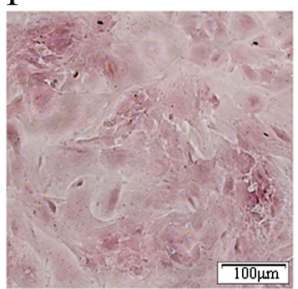

\section{IL-27 enhanced the regulation of hPMSCs on inducing the generation of Th1 and Th2 subsets from activated PBMCs}

Results from FCM analysis indicated that hPMSCs promoted the differentiation of $\mathrm{CD}^{+} \mathrm{T}$ cells into Th2 subsets $(P<0.01$; Fig. 4b, d) while inhibiting the differentiation into Th1 subsets
$(P<0.05$; Fig. 4a, d), as compared to PHA activated T cells. Moreover, these regulatory effects of hPMSCs on Th1 and Th2 subset generations were enhanced after hPMSCs were pretreated with IL-27 (Th1: $P<0.05$, Th2: $P<0.01$; Fig. 4a, b, d). Our results also showed that PDL1 can significantly enhance hPMSCs' regulatory effects on Th1 $(P<0.05)$ and Th2 $(P<0.05$; Fig. 4a, b, d) subset generations.

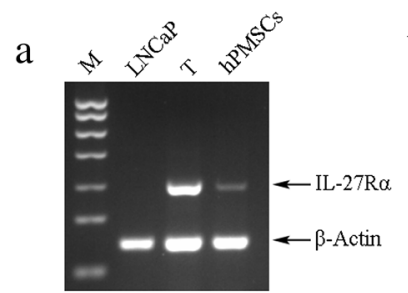

b

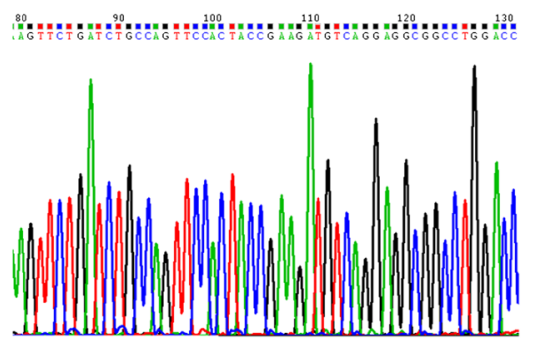

d

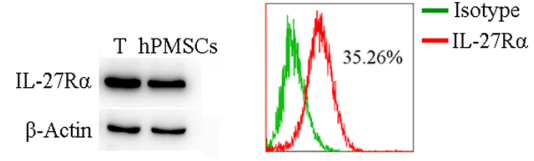

$$
\text { e }
$$

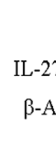

Fig. 2 IL-27R $\alpha$ expression in hPMSCs. a Detection of IL-27R $\alpha$ mRNA expression in $h P M S C s$ using RT-PCR; $\mathrm{CD}^{+} \mathrm{T}$ cells were used as a positive control and $L N C a P$ cells were used as a negative control. b, $\mathbf{c}$ Expression of IL-27R $\alpha$ mRNA expression in hPMSCs using gene
C

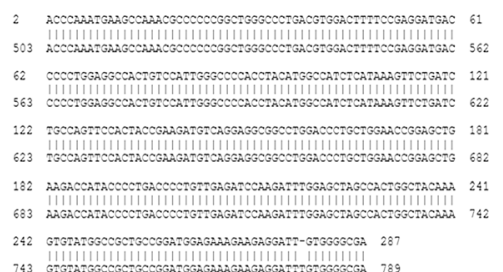

743.111111111111111111111111111111111111111111

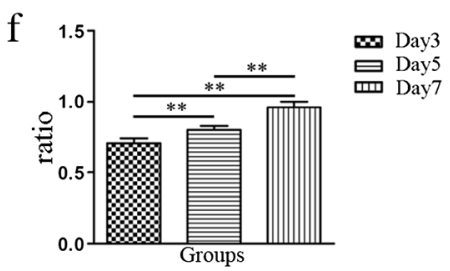

sequencing. d Detection of IL-27R $\alpha$ protein expression in hPMSCs using Western blot and flow cytometry analyses. e, f Expression of IL$27 \mathrm{R} \alpha$ as determined at different time points(3,5, and 7 days) from three passages of hPMSCs using Western blot 
a

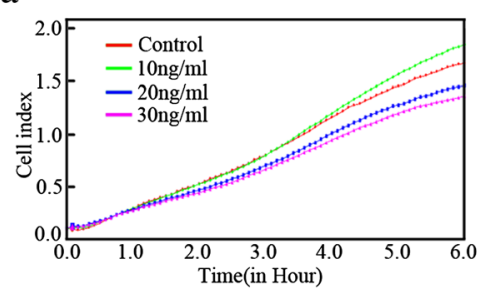

$\mathrm{b}$

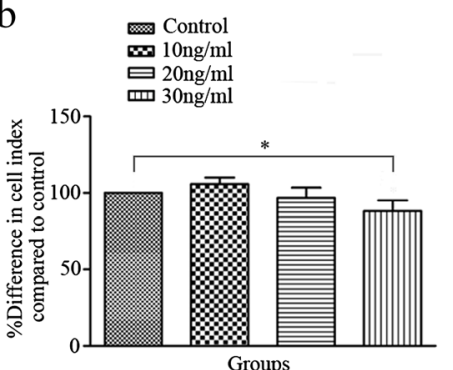

$\mathrm{c}$

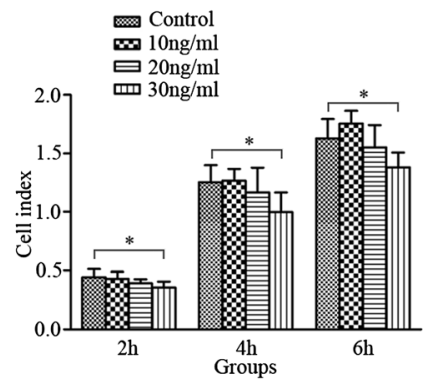

d

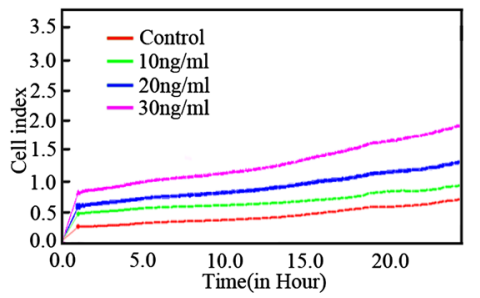

e

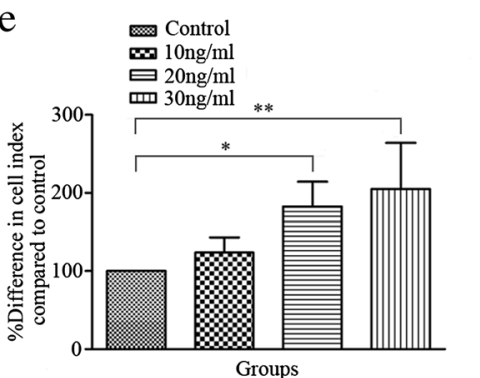

f

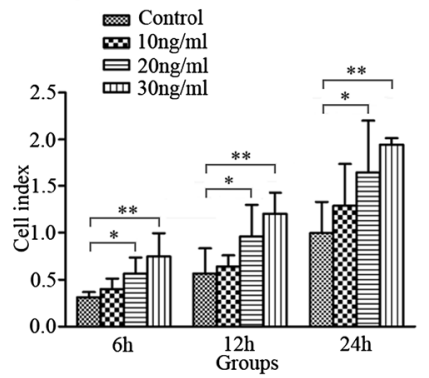

$\mathrm{g}$

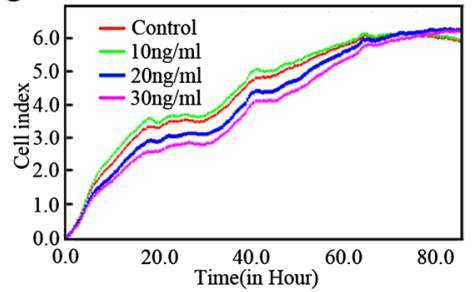

h
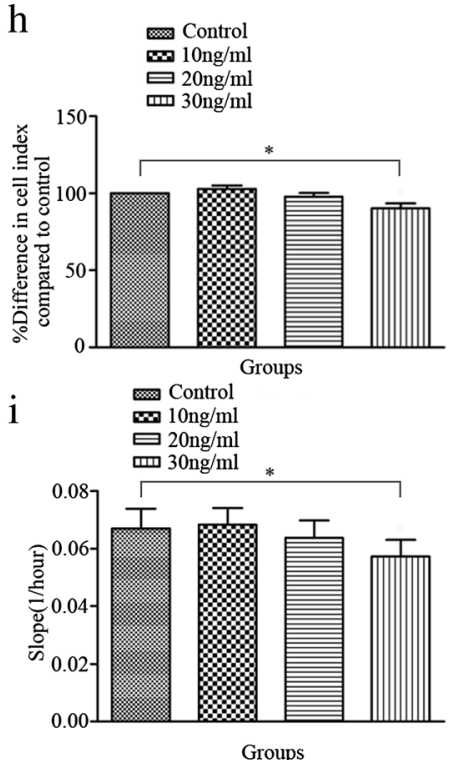

Fig. 3 Effects of IL-27 on adherence, migration and proliferation of hPMSCs. Representative figure of CI values for adherence (a), migration (d), and proliferation (g) of hPMSCs treated with/without IL27. Comparisons of the percent differences in the mean CI for adherence (b), migration (e), and proliferation (h) of hPMSCs treated with/without varying concentrations of IL-27. Representative figure on adherence (c)

\section{IL-27 upregulated the capacity for hPMSCs to induce IL-10 from CD4 ${ }^{+} \mathrm{T}$ cells}

Findings from our FCM analysis revealed that hPMSCs could induce secretion of IL-10 from PHA-activated $\mathrm{CD}^{+} \mathrm{T}$ cells $(P<0.01$; Fig. $4 \mathrm{c}, \mathrm{e})$. In response to IL-27 stimulation, hPMSCs induced significantly increased IL-10 secretion from CD4 ${ }^{+}$T cells $(P<0.05$; Fig. 4c, e). However, when the PDL1 blocking antibody was combined with hPMSCs, IL-10 secretion from $\mathrm{CD}^{+} \mathrm{T}$ cells was significantly decreased $(P<0.05$; Fig. $4 \mathrm{c}, \mathrm{e})$.

\section{IL-27 upregulated PDL1 expression in hPMSCs}

Results from a previous study in our laboratory revealed that PDL1 regulated the immunosuppression of hPMSCs on T cells [21]. In this study, PDL1 expression levels in hPMSCs were measured at $12,24,48$ or $72 \mathrm{~h}$ after incubation with IL27 at doses of $0,10,20$, or $30 \mathrm{ng} / \mathrm{ml}$. For all concentrations tested, IL-27 upregulated PDL1 expression in hPMSCs as compared with the $0 \mathrm{ng} / \mathrm{ml}$ group. In particular, hPMSCs and migration (f) CI values for hPMSCs at different time points following treatment with the indicated concentrations of IL-27. The rate of proliferation (i) was measured by analyzing the slope of the line between 0 and $80 \mathrm{~h}$ after IL-27 treatment or not in hPMSCs. The results presented are from three independent experiments $\left({ }^{*} P<0.05\right.$, $* * P<0.01)$

incubated with IL-27 at $20 \mathrm{ng} / \mathrm{ml}$ for $24 \mathrm{~h}$ induced maximal levels of PDL1 expression (Fig. 5a, b). Further analysis revealed enhanced expression levels of P-STAT1 in hPMSCs after stimulation with IL-27 for $1 \mathrm{~h}(P<0.01$; Fig. $5 \mathrm{c}$, d). After pretreatment with the JAK1/2 inhibitor-NCB018424, P-STAT1 expression levels were significantly reduced in hPMSCs treated with IL-27 for $1 \mathrm{~h}(P<0.05$; Fig. 5e, f). Moreover, expression levels of PDL1 in hPMSCs incubated with IL-27 for $24 \mathrm{~h}$ were significantly decreased when pretreated with the JAK1/2 inhibitor-INCB018424 for $1 \mathrm{~h}$ (Fig. 5g, h).

\section{Discussion}

MSCs have become a significant area of research focus in clinical immunology due to their capacity to oppose immunomodulatory effects and their biological characteristics of low immunogenicity. MSCs can regulate a number of biological functions in a variety of immune cells [27-30]. Their inhibitory effects upon $\mathrm{T}$ cells are of interests for use in the 
Fig. 4 IL-27 enhanced the regulatory effects of hPMSCs on the generation of Th1 and Th2 cells from activated $\mathrm{T}$ cells and induced IL-10 secretion from CD4 ${ }^{+} \mathrm{T}$ cells. PHA-activated PBMCs and hPMSCs were cocultured with or without PDL1 blocking antibodies. Th1 $\left(\mathrm{CD} 4^{+} \mathrm{IFN}-\gamma^{+} \mathrm{T}\right)$, Th2 $\left(\mathrm{CD} 4^{+} \mathrm{IL}-\right.$ $4^{+} \mathrm{T}$ ) cell subsets, and IL-10 secreting $\mathrm{CD} 4^{+} \mathrm{T}$ cells were measured with use of FCM. a-c FCM figure for Th1 and Th2 cell subsets and IL-10 secreting $\mathrm{CD} 4^{+} \mathrm{T}$ cells. d, e Th1 and Th2 cell subsets and secretion of IL-10 from $\mathrm{CD}^{+} \mathrm{T}$ cells (mean $\pm \mathrm{SD}$ ). The results presented are from three independent experiments $(* P<0.05, * * P<0.01)$ a

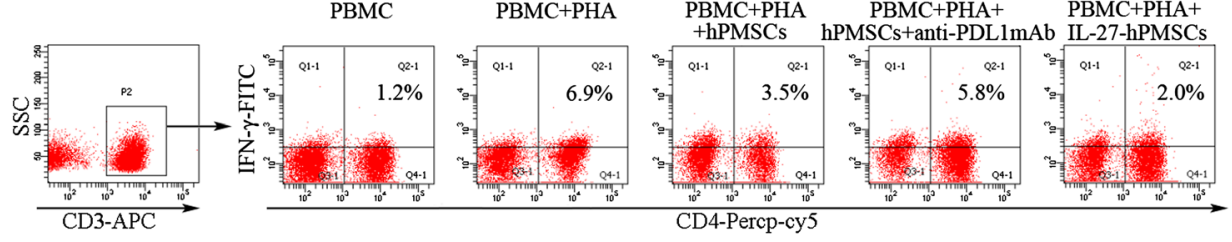

b

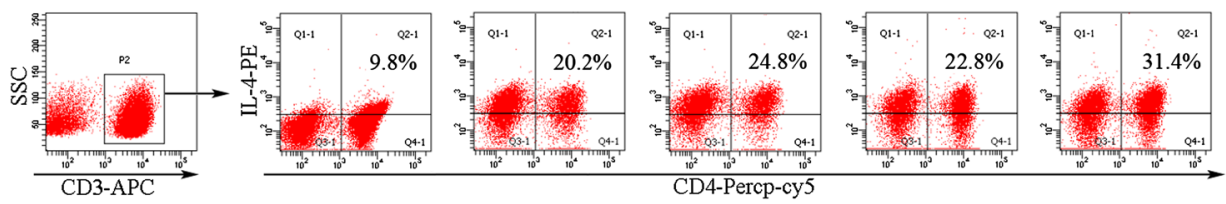

c

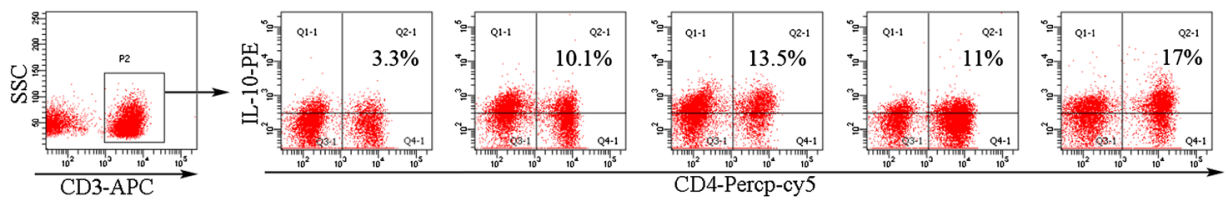

d

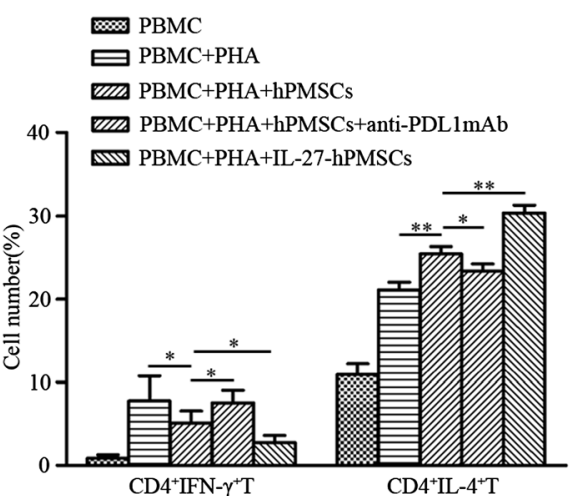

e $\quad$ PBMC

曰 $\mathrm{PBMC}+\mathrm{PHA}$

PBMC+PHA+hPMSCs

$\mathrm{PBMC}+\mathrm{PHA}+\mathrm{hPMSCs}+$ anti-PDL $1 \mathrm{mAb}$

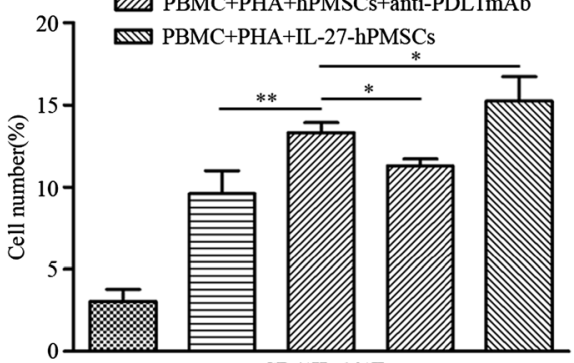

$\mathrm{CD} 4^{+} \mathrm{IL}-10^{+} \mathrm{T}$ prevention and treatment of autoimmune diseases [31, 32]. MSCs can be isolated from bone marrow, fat, placenta, skin, and other tissues [11]. We found that placental tissue contains high levels of MSCs, which have similar immunomodulatory effects as those derived from bone marrow, including negative effects upon regulating the activation and proliferation of $\mathrm{T}$ cells [19].

IL-27 is predominantly produced from activated macrophages and dendritic cells, which can exert either positive or negative regulatory effects upon immune responses. It has been reported that IL-27 can accelerate the progression of arthritis by promoting Th1 cell generation; however, IL-27 can also alleviate arthritis by suppressing Th17 responses [33]. In addition, IL-27 has also been shown to upregulate the expression of PDL1 in T cells and, in this way, negatively regulate $\mathrm{T}$ cell immune responses [6]. Previous results from our laboratory have revealed that hPMSCs inhibit T cell activation and proliferation through stimulating PDL1 expression [21]. Besides, the immunomodulatory effects of hPMSCs could also be affected by cytokine. However, it is unclear whether IL-27 affects immune regulatory effects of hPMSCs. Based upon results obtained from RT-PCR, gene sequencing, Western blot, and FCM assays, we found that
IL-27R $\alpha$ is expressed in hPMSCs; and findings from a related report have indicated that gp130, which is another subunit of the IL-27 receptor, is also expressed in placenta tissue [4]. Taken together, the results of our current experiment suggest that the biological functions of hPMSCs may be affected by IL-27 during immune responses.

In response to inflammation, hPMSCs can migrate to damaged tissue for activation of the immune response, whereas cytokines affect biological functions of hPMSCs. It has been reported that the inflammatory cytokines, IL- $1 \beta$ and TNF- $\alpha$, can affect the migration of MSCs [34]. However, it is unclear whether IL-27 also affects hPMSC migration. In this study, we first examined whether the adherence, migration, and proliferation of hPMSCs were affected by IL-27 as determined with use of RTCA. With this assay, we found that IL-27 at a concentration of $30 \mathrm{ng} / \mathrm{ml}$ significantly inhibited adherence and proliferation, whereas IL-27 at both 20 and $30 \mathrm{ng} / \mathrm{ml}$ promoted hPMSC migration. These results suggest that IL-27 can regulate the biological activity of hPMSCs.

Findings from recent studies have indicated that BMSCs can alleviate the symptoms of EAE by regulating Th1 and Th2 responses and promoting IL-10 secretion [14]. However, the immunosuppressive effects of hPMSCs as regulated by IL-27 
Fig. 5 IL-27 upregulated PDL1 expression in hPMSCs via the JAK/STAT1 pathway. a, b IL-27 upregulated PDL1 expression in hPMSCs. $\mathbf{c}, \mathbf{d}$ Detection of PSTAT1 and STAT1 expressions in IL-27 $(20 \mathrm{ng} / \mathrm{ml})$-treated hPMSCs for $0,10,20,30,60$, or $120 \mathrm{~min}$ as determined with use of Western blot. e, f P-STAT1 and STAT1 protein levels in IL-27-treated hPMSCs for $1 \mathrm{~h}$, pretreated with/without the JAK1/2 inhibitor (INCB018424) for $1 \mathrm{~h} . \mathbf{g}, \mathbf{h}$ Expression of PDL1 in IL-27treated hPMSCs for $24 \mathrm{~h}$, which were pretreated with/without the JAK1/2 inhibitor (INCB018424) using FCM analysis. The results presented are from three independent experiments $(* P<0.05, * * P<0.01)$ a

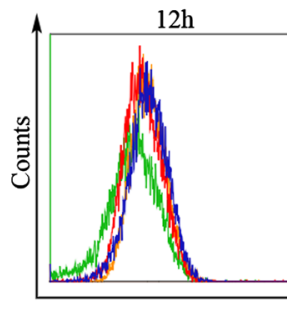

b

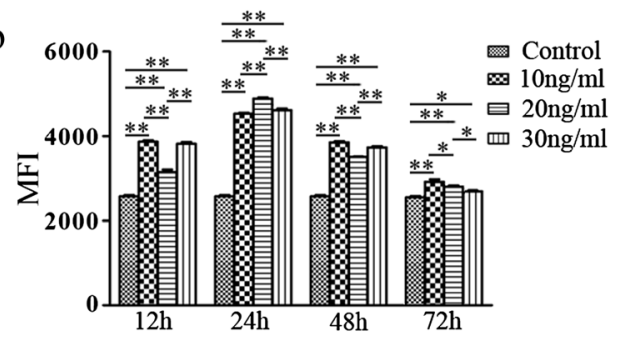

C

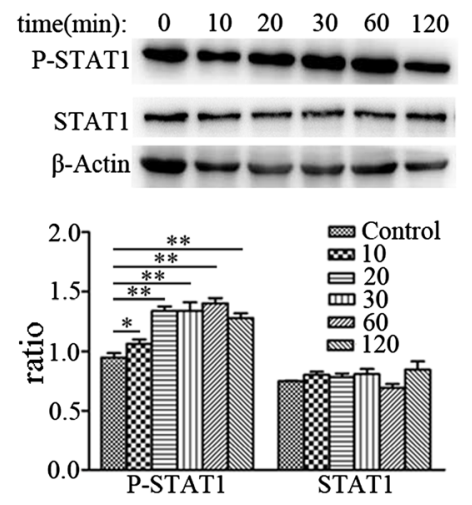

$\mathrm{g}$

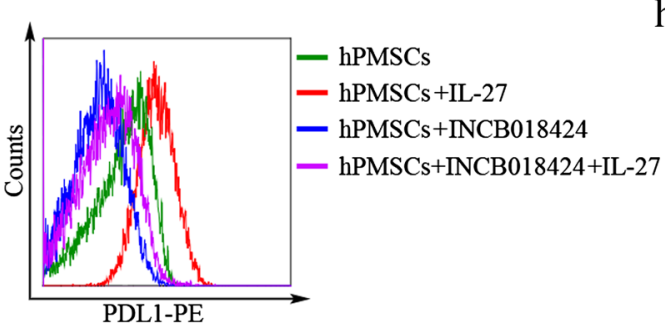

$48 \mathrm{~h}$

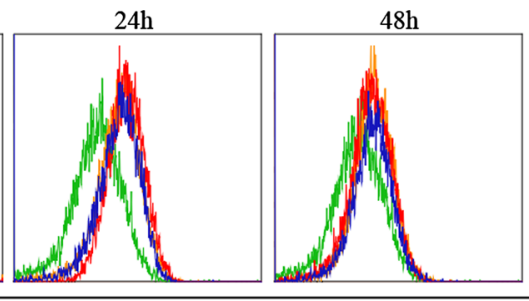

$72 \mathrm{~h}$

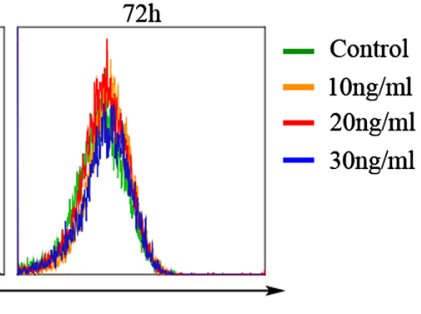

e

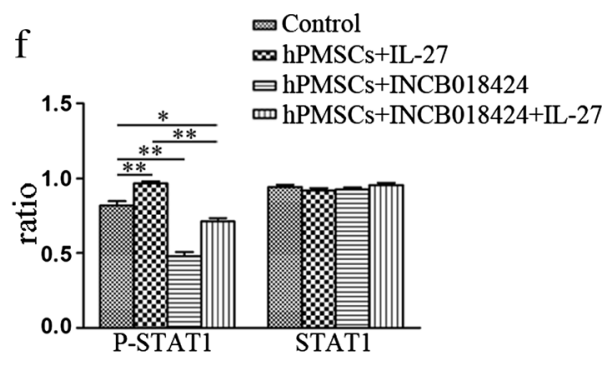

m Control

$\infty \mathrm{hPMSCs}+\mathrm{IL}-27$

$\square \mathrm{hPMSCs}+\mathrm{INCB} 018424$

๓m hPMSCs+INCB018424+IL-27

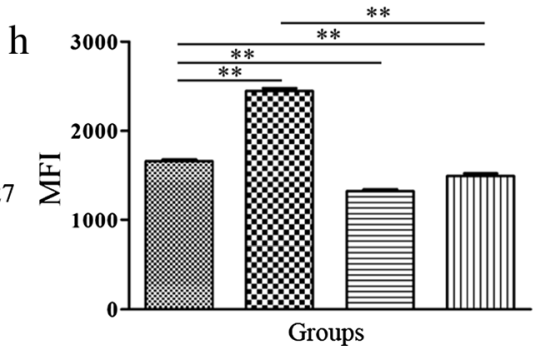

remain to be determined. In this study, we found that IL-27 can promote the effects of hPMSCs in enhancing the differentiation of PHA-activated T cells into Th2 subsets, IL-10 secretion from $\mathrm{CD}^{+} \mathrm{T}$ cells, and inhibition of Th1 cell generation by hPMSCs. These results are consistent with previous findings indicating that IFN- $\gamma$ and TNF- $\alpha$ can regulate the inhibitory effects of hPMSCs on T cell function [17]. In this way, an inverse relationship exists between hPMSCs and inflammatory factors. While hPMSCs can regulate inflammatory factors secreted from immune cells, their immunomodulatory effects are regulated by inflammatory cytokines. Therefore, when applying hPMSCs in the treatment of inflammatory diseases, inflammatory factor levels in patients may affect the clinical outcome of hPMSCs.

PDL1, also known as B7H1/CD274, is an important regulatory molecule involved with inhibiting $\mathrm{T}$ cell immune responses. Previous findings from our laboratory have shown that PDL1 plays an important role in the regulation of hPMSCs on T cell responses [21]. Based on that study, we blocked the expression of PDL1 in hPMSCs using PDL1blocking antibodies to investigate their molecular mechanisms. With this protocol, we found that the capacity for hPMSCs to induce the generation of Th1 or Th2 and IL-10 secretion from $\mathrm{CD}^{+} \mathrm{T}$ cells was partially reversed, suggesting an involvement of PDL1 in this process. Based on these results, we next examined the effect of IL-27 on the expression of PDL1 in hPMSCs. Our current results demonstrate that IL27 can upregulate PDL1 expression in hPMSCs. Further work involved with examining the potential molecular mechanisms of these effects has revealed that IL-27-treated hPMSCs have higher expressions of P-STAT1. With the addition of the JAK1/2 inhibitor, expressions of both P-STAT1 and PDL1 
in hPMSCs were significantly decreased. Taken together, these findings indicate that IL-27 upregulates PDL1 levels in hPMSCs through the JAK/STAT1 signaling pathway.

In conclusion, our current results demonstrate that (1) IL$27 \mathrm{R} \alpha$ expression is present in hPMSCs, (2) IL-27 exerts inhibitory effects upon hPMSC adherence and proliferation, and (3) IL-27 promotes migration of hPMSCs. In addition, our results also indicate that IL-27 can upregulate PDL1 expression in hPMSCs through the JAK/STAT1 pathway. Such effects enhance the regulatory effects of hPMSCs on Th1 and $\mathrm{Th} 2$ cell differentiations and IL- 10 secretion from $\mathrm{CD} 4^{+} \mathrm{T}$ cells. These findings provide new and important insights into understanding the mechanism of hPMSCs for potential use in clinical cell therapies.

Acknowledgements This study was supported by grants from the National Natural Science Foundation of China (31370905, 31540015).

Compliance with ethical standards The project was approved by the Ethics Committee of the Affiliated Hospital of Binzhou Medical College, Yantai, and informed consent was obtained from all sample donors.

Conflict of interest The authors declare that they have no conflict of interest.

Open Access This article is distributed under the terms of the Creative Commons Attribution 4.0 International License (http:// creativecommons.org/licenses/by/4.0/), which permits unrestricted use, distribution, and reproduction in any medium, provided you give appropriate credit to the original author(s) and the source, provide a link to the Creative Commons license, and indicate if changes were made.

\section{References}

1. Yoshida H, Hunter CA. The immunobiology of interleukin-27. Annu Rev Immunol. 2015;33:417-43.

2. Li JP, Wu H, Xing W, Yang SG, Lu SH, Du WT, et al. Interleukin27 as a negative regulator of human neutrophil function. Scand J Immunol. 2010;72(4):284-92.

3. Alejandro VV, Larkin J, Saris CJ, Caton AJ, Lucas S, Wong T, et al. Positive and negative regulation of the IL-27 receptor during lymphoid cell activation. J Immunol. 2005;174(12):7684-91.

4. Coulomb-L'Herminé A, Larousserie F, Pflanz S, Bardel E, Kastelein RA, Devergne O. Expression of interleukin-27 by human trophoblast cells. Placenta. 2007;28(12):1133-40.

5. Erices A, Conget P, Rojas C, Minguell JJ. gp130 activation by soluble interleukin-6 receptor/interleukin- 6 enhances osteoblastic differentiation of human bone marrow-derived mesenchymal stem cells. Exp Cell Res. 2002;280(1):24-32.

6. Iwasaki Y, Fujio K, Okamura T, Yamamoto K. Interleukin-27 in T cell immunity. Int J Mol Sci. 2015;16(2):2851-63.

7. Belle L, Agle K, Zhou V, Yin-Yuan C, Komorowski R, Eastwood $\mathrm{D}$, et al. Blockade of interleukin-27 signaling reduces GVHD in mice by augmenting Treg reconstitution and stabilizing Foxp3 expression. Blood. 2016;128(16):2068-82.

8. Fitzgerald DC, Ciric B, Touil T, Harle H, Grammatikopolou J, Sarma JD, et al. Suppressive effect of IL-27 on encephalitogenic
Th17 cells and the effector phase of experimental autoimmune encephalomyelitis. J Immunol. 2007;179(5):3268-75.

9. Andrews C, McLean MH, Durum SK. Interleukin-27 as a novel therapy for inflammatory bowel disease: a critical review of the literature. Inflamm Bowel Dis. 2016;22:2255-64.

10. Liao W, Pham V, Liu L, Riazifar M, Pone EJ, Zhang S, et al. Mesenchymal stem cells engineered to express selectin ligands and IL-10 exert enhanced therapeutic efficacy in murine experimental autoimmune encephalomyelitis. Biomaterials. 2016;77: 87-97.

11. Wang Y, Chen X, Cao W, Shi Y. Plasticity of mesenchymal stem cells in immunomodulation: pathological and therapeutic implications. Nat Immunol. 2014;15(11):1009-16.

12. Zhang Z, Han Y, Song J, Luo R, Jin X, Mu D, et al. Interferon- $\gamma$ regulates the function of mesenchymal stem cells from oral lichen planus via indoleamine 2,3-dioxygenase activity. J Oral Pathol Med. 2015;44(1):15-27.

13. Geng L, Li X, Feng X, Zhang J, Wang D, Chen J, et al. Association of TNF- $\alpha$ with impaired migration capacity of mesenchymal stem cells in patients with systemic lupus erythematosus. J Immunol Res. 2014;2014:169082.

14. Wang D, Li SP, Fu JS, Bai L, Guo L. Resveratrol augments therapeutic efficiency of mouse bone marrow mesenchymal stem cellbased therapy in experimental autoimmune encephalomyelitis. Int J Dev Neurosci. 2016;49:60-6.

15. Casiraghi F, Azzollini N, Cassis P, Imberti B, Morigi M, Cugini D, et al. Pretransplant infusion of mesenchymal stem cells prolongs the survival of a semiallogeneic heart transplant through the generation of regulatory T cells. J Immunol. 2008;181(6):3933-46.

16. Hegyi B, Kudlik G, Monostori É, Uher F. Activated T-cells and proinflammatory cytokines differentially regulate prostaglandin E2 secretion by mesenchymal stem cells. Biochem Biophys Res Commun. 2012;419(2):215-20.

17. Li H, Wang W, Wang G, Hou Y, Xu F, Liu R, et al. Interferongamma and tumor necrosis factor-alpha promote the ability of human placenta-derived mesenchymal stromal cells to express programmed death ligand- 2 and induce the differentiation of CD $4^{+}$interleukin- $10^{+}$and $\mathrm{CD} 8^{+}$interleukin- $10^{+}$Treg subsets. Cytotherapy. 2015;17(11):1560-71.

18. Sheng H, Wang Y, Jin Y, Zhang Q, Zhang Y, Wang L, et al. A critical role of IFN- $\gamma$ in priming MSC-mediated suppression of T cell proliferation through up-regulation of B7-H1. Cell Res. 2008;18(8):846-57.

19. Luan X, Li G, Wang G, Wang F, Lin Y. Human placenta-derived mesenchymal stem cells suppress $\mathrm{T}$ cell proliferation and support the culture expansion of cord blood CD $34^{+}$cells: a comparison with human bone marrow-derived mesenchymal stem cells. Tissue Cell. 2013;45(1):32-8.

20. Chinnadurai R, Copland IB, Patel SR, Galipeau J. IDOindependent suppression of $\mathrm{T}$ cell effector function by IFNgamma-licensed human mesenchymal stromal cells. J Immunol. 2014;192(4):1491-501.

21. Wang GY, Zhang SY, Wang FF, Li GY, Zhang LX, Luan XY. Expression and biological function of programmed death ligands in human placenta mesenchymal stem cells. Cell Biol Int. 2013;37(2):137-48.

22. Wang G, Wang F, Li H, Zhang L, Luan X. PD-L1/PD-L2 on human placenta-derived mesenchymal stem cells inhibits the IL-17 secretion of peripheral blood T cells. Chin J Cell Mol Immunol. 2013;29(2):132-6.

23. Hunter CA. New IL-12-family members: IL-23 and IL-27, cytokines with divergent functions. Nat Rev Immunol. 2005;5(7):52131.

24. Di Carlo E, Sorrentino C, Zorzoli A, Di Meo S, Tupone MG, Ognio E, et al. The antitumor potential of interleukin-27 in prostate cancer. Oncotarget. 2014;5(21):10332-41. 
25. Dowling CM, Herranz Ors C, Kiely PA. Using real-time impedance-based assays to monitor the effects of fibroblast-derived media on the adhesion, proliferation, migration and invasion of colon cancer cells. Biosci Rep. 2014;34(4):415-32.

26. Li H, Wang G, Wang W, Wang F, Liu R, Luan X. Regulatory effects of IFN- $\gamma$ on the expression of PDL2 on human placental mesenchymal stem cells (hPMSCs) and the hPMSCs-induced differentiation of peripheral blood $\mathrm{CD} 8^{+} \mathrm{IL}-10^{+} \mathrm{T}$ cell subsets. Chin J Cell Mol Immunol. 2014;34:691-6.

27. Qu M, Cui J, Zhu J, Ma Y, Yuan X, Shi J, et al. Bone marrowderived mesenchymal stem cells suppress NK cell recruitment and activation in PolyI: C-induced liver injury. Biochem Biophys Res Commun. 2015;466(2):173-9.

28. Hackstein H, Tschipakow I, Bein G, Nold P, Brendel C, Baal N. Contact-dependent abrogation of bone marrow-derived plasmacytoid dendritic cell differentiation by murine mesenchymal stem cells. Biochem Biophys Res Commun. 2016;476(1):15-20.

29. Chen W, Huang Y, Han J, Yu L, Li Y, Lu Z, et al. Immunomodulatory effects of mesenchymal stromal cellsderived exosome. Immunol Res. 2016;64(4):831-40.
30. Bonnaure G, Gervais-St-Amour C, Néron S. Bone marrow mesenchymal stem cells enhance the differentiation of human switched memory B lymphocytes into plasma cells in serum-free medium. Immunol Res. 2016;2016:7801781.

31. Malcherek G, Jin N, Hückelhoven AG, Mani J, Wang L, Gern U, et al. Mesenchymal stromal cells inhibit proliferation of virusspecific CD8 ${ }^{+}$T cells. Leukemia. 2014;28(12):2388-94.

32. Fujino M, Zhu P, Kitazawa Y, Chen JM, Zhuang J, Li XK. Mesenchymal stem cells attenuate rat graft-versus-host disease. Animal Models Stem Cell Therapy. 2014;1213:341-53.

33. Meka RR, Venkatesha SH, Dudics S, Acharya B, Moudgil KD. IL27-induced modulation of autoimmunity and its therapeutic potential. Autoimmun Rev. 2015;14(12):1131-41.

34. Ries C, Egea V, Karow M, Kolb H, Jochum M, Neth P. MMP-2, MT1-MMP, and TIMP-2 are essential for the invasive capacity of human mesenchymal stem cells: differential regulation by inflammatory cytokines. Blood. 2007;109:4055-63. 